\title{
ABSORCIÓN DE DOS FOTONES EN POZOS CUÁNTICOS FORMADOS CON GaAs DOPADO CON ALUMINIO
}

\author{
$\checkmark$ CARlos Andrés Álvarez OCAMPO ${ }^{1}$ \\ RODRIGo ACUÑA HERRERA ${ }^{2}$
}

\section{RESUMEN}

En este trabajo mostramos como la absorción de dos fotones es modificada debido a la fracción molar de aluminio dopante presente en los pozos cuánticos formados con arseniuro de galio. Se realizó el estudio teniendo en cuenta el índice de refracción que depende de la fracción molar de aluminio dopante y de la longitud de onda de la luz que incide sobre los pozos. Los resultados sugieren como manipular los parámetros de diseño para mejora el desempeño de un dispositivo foto-detector idóneo para longitudes de onda de $1.310 \mathrm{~nm}$ y $1.550 \mathrm{~nm}$.

PALABRAS CLAVES: Absorción de dos fotones, Pozos cuánticos, Fracción molar, Portadores de carga, Telecomunicaciones ópticas, Gap de energía.

\section{ABSORPTION OF TWO PHOTONS IN QUANTUM WELLS FORMED WITH ALUMINUM DUMPED GaAs}

\section{ABSTRACT}

In this work we show how the absorption of two photons is modified due to the molar fraction of aluminum dopant present in the quantum wells formed with gallium arsenide. The study was performed taking into account the refractive index that depends on the mole fraction of aluminum dopant and the wavelength of light that hits the wells. The results suggest how to manipulate the design parameters to improve the performance of a photo-detector device suitable for wavelengths of $1.310 \mathrm{~nm}$ and $1.550 \mathrm{~nm}$.

KEYWORDS: Two photon absortion, quantum Wells, molar fraction, Charge Carrier, Optical telecommunication, Energy gap.

1 Ingeniero Físico. Universidad Nacional de Colombia, sede Medellín. Docente Universidad de Medellín, Colombia.

2 Doctorado Ingeniería Eléctrica, Universidad de Wisconsin, Estados Unidos. Maestría en Física. Universidad Nacional de Colombia. Docente Universidad Nacional de Colombia, Medellín, Colombia.

Autor de correspondencia: Álvarez Ocampo, C.A. (Carlos Andrés): Universidad de Medellín, Medellín, Colombia. Correo electrónico: caalvarez@udem.edu.co

\section{Historia del artículo:}

Artículo recibido: 30-VI-2017/ Aprobado: 13-IX-2017

Disponible online: 30 de marzo de 2018

Discusión abierta hasta abril de 2019 


\section{ABSORÇÃO DE DOIS FÓTONS EM POÇOS QUÂNTICOS FORMADOS COM GaAs DUMPADOS DE ALUMÍNIO}

\section{RESUMO}

Neste trabalho como absorção de dois fotões é modificado devido à fracção de moles de dopante de alumínio presente nos poços formados quânticos de arsenieto de gálio. o estudo considerando o índice de refracção depende da fracção de moles de dopante de alumínio e o comprimento de onda da luz incidente sobre os poços foi realizada. Os resultados sugerem como manipular os parâmetros de concepção para melhorar o desempenho de um dispositivo fotodetector adequado para comprimentos de onda $1.310 \mathrm{~nm}$ e $1.550 \mathrm{~nm}$.

PALABRAS-CHAVE: Absorção de dois fótons, Poços quânticos, Fração molar, Carregador de carga, Telecomunicação óptica, Espaço de energia.

\section{INTRODUCCIÓN}

Los pozos cuánticos son estructuras en capas delgadas de semiconductores. Obtienen la mayor parte de sus propiedades especiales gracias al confinamiento cuántico de portadores de carga. Pueden ser hechos con un alto grado de precisión con técnicas de crecimiento de películas delgadas como el crecimiento epitaxial por múltiples haces moleculares (MBE). Los pozos cuánticos a partir de estructuras semiconductoras poseen distribuciones de bandas que conforman un pozo de potencial, es importante destacar que, para los pozos cuánticos, los electrones en la banda de conducción, y los huecos en la banda de valencia se comportan como partículas con masas eficaces diferentes de la masa del electrón libre (Colace et al., 2006).

Los pozos cuánticos pueden ser utilizados para dispositivos electrónicos avanzados (por ejemplo, transistores de efecto campo modulado por dopados, transistores bipolares de hetero unión, dispositivos de efecto túnel resonante), componentes ópticos (por ejemplo, guías de onda, espejos, microresonadores), y dispositivos opto-electrónicos (por ejemplo, diodos láser, foto-detectores y super redes ópticas (Adachi, 1985). Hay al menos dos razones para hacer integración, en primer lugar, la ganancia electrónica permite que los requisitos de energía óptica se reduzcan gracias al aprovechamiento de la absorción de dos fotones (2PA) podremos evitar la etapa de amplificación; la segunda razón es que la electrónica es muy buena en la realización de funciones lógicas complejas a nivel local (Ren et al., 2011). Combinando electrónica con las habilidades de la óptica para la interconexión podemos tener lo mejor de ambos mundos. Otro enfoque para tal integración es combinar dispositivos de pozo cuántico con silicio como puente a la electrónica (Villeneuve et al., 1995).

La absorción de dos fotones (2PA) es un proceso en el que interviene dos campos de radiación que interactúa con portadores de carga que se excitan y des-excitan en periodos de tiempo del orden de femtosegundos, en óptica no lineal se le conoce como fenómeno no paramétrico, en el que intervienen una banda virtual entre la banda de conducción y la de valencia, este proceso se describe a través de la parte imaginaria de la susceptibilidad de tercer orden $\left(x^{(3)}\right)$ del material, adicionalmente el material retiene energía procedente de los campos de radiación, por tanto la energía podría no conservarse y existir efectos térmicos adicionales (Wagner, 2007). El arreglo de múltiples pozos cuánticos maximiza la posibilidad que existan efectos ópticos no lineales debidos a la susceptibilidad de tercer orden. 


\section{MARCO TEÓRICO}

2PA ocurre de dos maneras, cuando se absorben fotones que tienen la misma energía (D2PAcaso degenerado) y cuando sus energías no son las mismas (ND2PA-caso no degenerado), la condición necesaria que deben cumplir los dos fotones es que los aportes de energías de ambos sumen como mínimo la energía del gap entre la capa de valencia y la de conducción en el pozo, según

$$
E_{g} \leq \hbar \omega_{1}+\hbar \omega_{2}
$$

adicionalmente los dos fotones pueden tener el mismo estado de polarización (TM-TM o TE-TE) o diferente (TE-TM); en el caso ND2PA un fotón puede aportar hasta el 90\% de la energía del gap, esta situación se conoce como absorción de dos fotones extremadamente no degenerada (END-2PA) (Fishman et al., 2011).

Para obtener los coeficientes 2PA para cada caso de polarización y energía de cada fotón, se usaron diferentes fracciones molares en la concentración de aluminio y se calculó usando el software MATLAB solo para los casos donde los dos fotones tienen igual estado de polarización.

La energía de band gap en el pozo cuántico depende de la fracción molar de aluminio presente, como lo muestra la siguiente ecuación (Chuang, 2009):

$$
W=\frac{2 \pi}{\hbar}\left(\frac{e E_{1}}{2 m \omega_{1}}\right)^{2}\left(\frac{e E_{2}}{2 m \omega_{2}}\right)^{2} \sum_{F}\left|\sum_{I} \frac{M_{F I}^{(2)} M_{I O}^{(1)}}{E_{I}-E_{O}-\hbar \omega_{1}}+\frac{M_{F I}^{(1)} M_{I O}^{(2)}}{E_{I}-E_{O}-\hbar \omega_{2}}\right|^{2} \delta\left(E_{I}-E_{O}-\hbar \omega_{1}-\hbar \omega_{2}\right)
$$

$E_{1}$ y $E_{2}$ son los campos de radiación de los fotones, $M_{X Y}^{(i)}$ son las matrices de transición del momento dipolar de los portadores de carga en el pozo para cada par de bandas y están gobernadas por probabilidades cuánticas, $E_{O}$ es la energía de la banda de valencia, $E_{I}$ es la energía de la banda virtual o intermedia que esta entre la banda de valencia y la de conducción. La sumatorio se realiza para cada posible transición de la banda de valencia (0) a la banda

$$
E_{g}(x)=1,424+1,266 x+0,26 x^{2}(\mathrm{eV})
$$

donde $x$ es fracción molar de aluminio presente en la capa semiconductora que actúa como pozo de potencial, claramente se aprecia que a mayor fracción molar la energía del gap aumenta, lo que nos supone que debemos usar una longitud de onda de prueba menor que nos garantice que se cumple con el límite de energía del gap, esta situación se programa de tal manera que escogiendo una fracción molar determinada y fijando la longitud de onda de bombeo, que fue en nuestro caso una señal viajando por fibra óptica que típicamente son a $1.310 \mathrm{~nm}$ y $1.550 \mathrm{~nm}$, con esta información podemos hacer un barrido para varias longitudes de onda que aportaran el porcentaje de energía faltante para completar el gap del pozo, para evitar el caso degenerado, la longitud de onda de prueba es mucho menor que la de bombeo fijada (menor a $1.000 \mathrm{~nm}$ ).

El coeficiente 2PA $\alpha_{2}$ se puede encontrar usando la siguiente ecuación (Xia et al., 2009):

$$
\alpha_{2}^{(2)}=\left(\frac{8 \pi \hbar \omega_{2}}{c n_{2} E_{2}^{2}}\right) \mathrm{W}
$$

donde $E^{2}=\frac{2 I}{n c \epsilon_{0}} \quad ; I=\frac{n c \epsilon_{0} \omega^{2} A_{0}^{2}}{2}$

Además, la tasa de transición 2PA es definida como sigue:

virtual o intermedia (I) y para cada transición de la banda intermedia la banda final (F) o de conducción.

El estudio del comportamiento del índice de refracción en la estructura se hace a partir del software COMSOL Multiphysics cuyo método de simulación son los elementos finitos, se busca el índice de refracción efectivo del conjunto de pozos cuánticos ya que debe ser tenido en cuenta para el cálculo de la absorción. Para los materiales que 
son combinación de elementos de los grupos III y $\mathrm{V}$ como el $\mathbf{A l}_{\mathbf{x}} \mathbf{G a}_{1-\mathbf{x}} \mathbf{A s}$ se puede aproximar el índice de refracción cuando se trata de energía por debajo del band gap como sigue:

$$
n(\omega) \approx \sqrt{\frac{\varepsilon^{\prime}(\omega)}{\varepsilon_{0}}}
$$

Donde $\varepsilon^{\prime}(\omega)$ es la parte real de la función permitividad, la parte imaginaria es despreciable para energías por debajo del band gap (Chuang, 2009).

$$
\begin{gathered}
\frac{\varepsilon^{\prime}(\omega)}{\varepsilon_{0}} \approx A(x)\left\{f(y)+\frac{1}{2}\left[\frac{E_{g}(x)}{E_{g}(x)+\Delta(x)}\right]^{\frac{3}{2}} f\left(y_{s o}\right)\right\}+B(x) \\
f(y)=\frac{1}{y^{2}}\left[2-(1+y)^{\frac{1}{2}}-(1-y)^{\frac{1}{2}}\right] \\
y=\frac{\hbar \omega}{E_{g}(x)} \\
y_{s o}=\frac{\hbar \omega}{\left[E_{g}(x)+\Delta(x)\right]}
\end{gathered}
$$

$E_{g}(x)$ Determina la energía del band gap según la Ecuación (2) y $\Delta(x)$ es la energía del acople spinorbit. Para el $\mathrm{Al}_{x} \mathrm{Ga}_{1-\mathrm{x}} \mathrm{As}$ los parámetros en función de la fracción molar $x$ son:

$$
\begin{gathered}
\Delta(x)=0,24-0,5 x(\mathrm{eV}) \\
A(x)=6,64+16,92 x \\
B(x)=9,2-9,22 x
\end{gathered}
$$

Es de notar que el índice de refracción es generalmente dispersivo (varia con la frecuencia) cerca o por encima del band gap con una rápida variación en los límites de energías para transición inter-bandas, además depende ligeramente del dopado en el semiconductor, de la temperatura y la deformación (Chuang, 2009) En nuestro caso de estudio no tenemos problema con la absorción lineal ya que usamos dos haces que por sí solos no la generan, el requisito es que ambos haces actúen al mismo tiempo para que se genere $2 \mathrm{PA}$.

Muestra estructura contiene 40 pozos intercalados entre barreras a lo que llamaremos periodos, esta región de pozos cuántico tiene un espesor 1,2 micrómetros, la estructura está sustentada por una oblea de arseniuro de galio y rodeada por aire. (Ver Figura 1). Para nuestro caso de estudio seleccionamos una geometría simple, ancho de pozo d=10nm y ancho de barreras de 20nm. (Ver Figura 2). La profundidad $w$ se vuelve importante en una etapa posterior de fabricación del dispositivo, la dirección $z$ se toma como la dirección en la que se depositan las capas de material, la polarización TM la definimos cuando el campo eléctrico $\vec{E}$ se encuentra paralelo a este eje $z$, mientras que la polarización TE cuando $\vec{E}$ es perpendicular al eje $z$ (Pattanaik et al., 2016). Usamos diferentes fracciones molares $x$ para dopar el pozo, en cada caso de fracción molar de aluminio en el pozo le corresponde una fracción molar 0,1 veces mayor en las barreras, por ejemplo, si en el pozo existe una fracción molar de aluminio de 0,2 , en las barreras la fracción es de 0,3.

Figura 1. Ilustración de la disposición de pozos simulada, A) estructura completa, B) zoom a la sección de un solo pozo
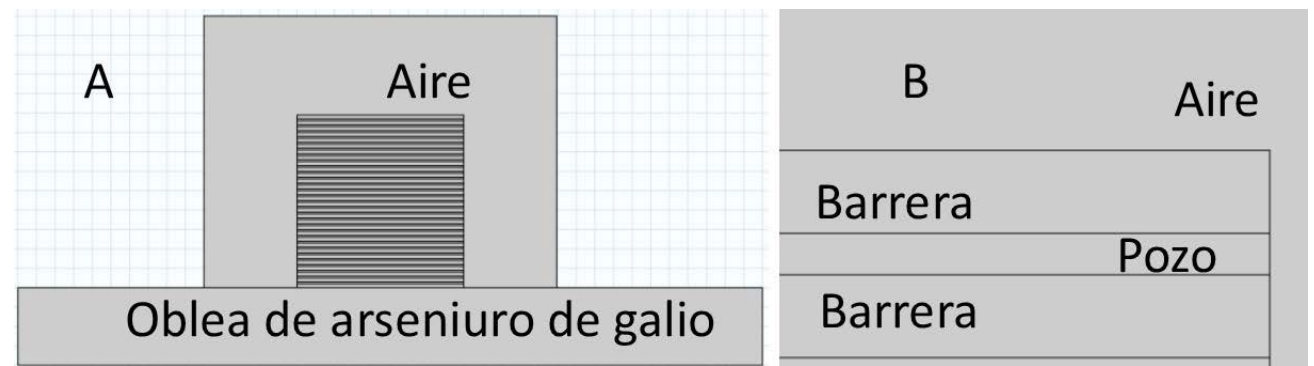
Figura 2. Ilustración de un guía de onda rectangular, la región con $\mu_{1} \epsilon_{1}$ posee mayor índice de refracción que las regiones marcadas con $\mu \epsilon$, por tanto, se configura un guía de onda.

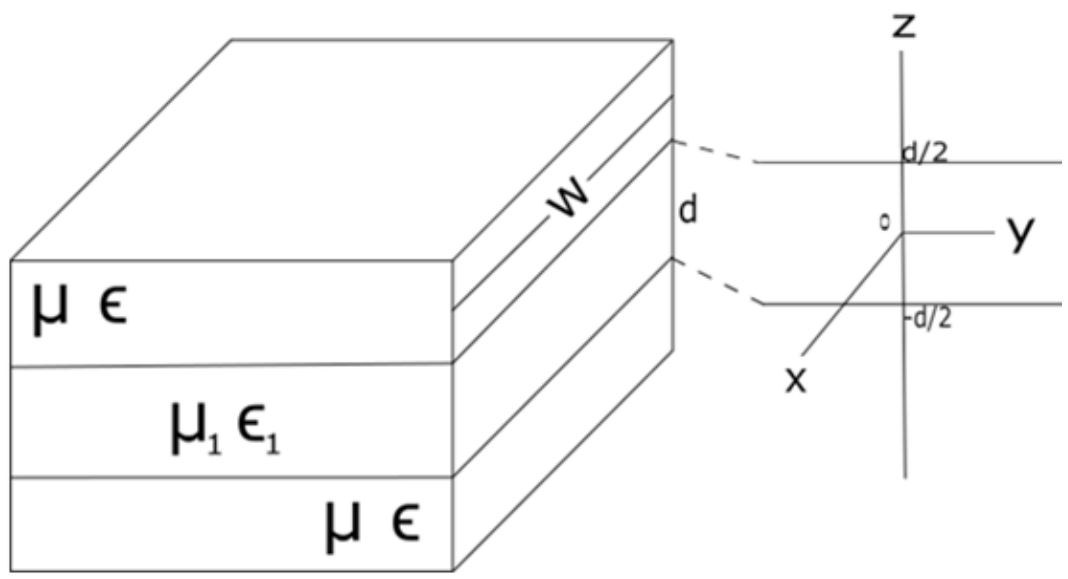

\section{RESULTADOS}

En las Figura 3 y 4 mostramos el coeficiente de 2PA para estados de polarización TM-TM y TE-TE respectivamente. Claramente, mostramos que existe fuerte dependencia con la fracción molar de aluminio presente en el pozo, nosotros solo presentamos tres casos de fracción molar ya que todos los casos estudiados muestran el mismo comportamiento, cuya tendencia es de disminuir el coeficiente 2PA conforme la fracción molar de aluminio en el pozo aumenta. Además, apreciamos que el caso TM-TM proporciona una mejora en los valores del coeficiente para las mismas condiciones de dopado de aluminio e ilumina- ción de los pozos. Esto es debido a que la polarización TM se considera en dirección paralela a la dirección de creciente del pozo, luego el vector de polarización del fotón es paralelo al vector momento del electrón confinado en el pozo, por tanto hay mayor probabilidad que el fotón y el electrón interactúen.

Este resultado para el coeficiente 2PA manifiesta una posibilidad de ser implementado en la detección de luz en sistemas de telecomunicaciones, específicamente la banda de $1.550 \mathrm{~nm}$, donde es importante la sensibilidad, la estructura propuesta en este trabajo es factible de fabricar con la tecnología actualmente conocida.

\section{Figura 3. Absorción de dos fotones, para polarización TM-TM, usando una longitud de onda de bombeo de $1.550 \mathrm{~nm}$.}

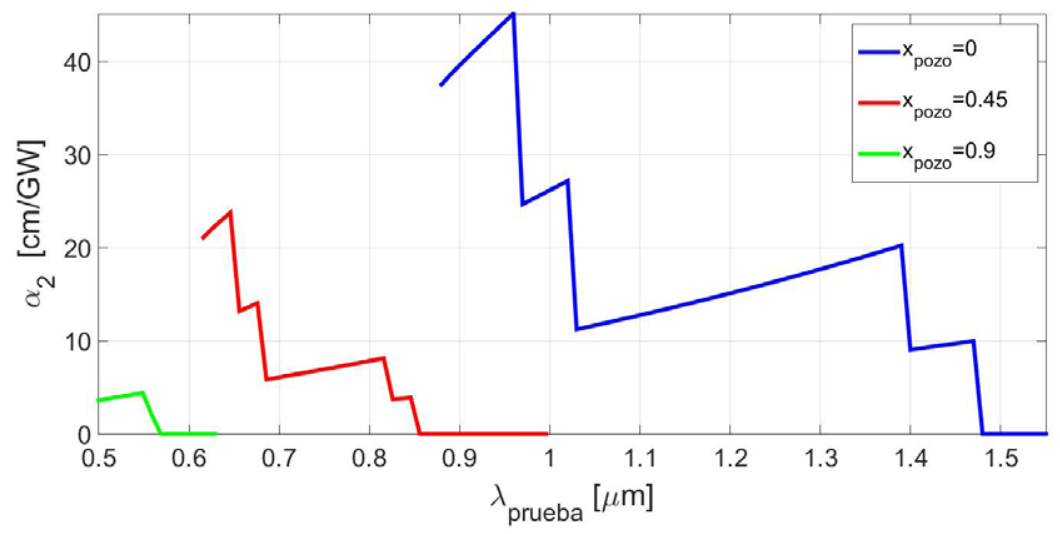


Figura 4. Absorción de dos fotones, para polarización TE-TE, usando una longitud de onda de bombeo de 1.550 nm

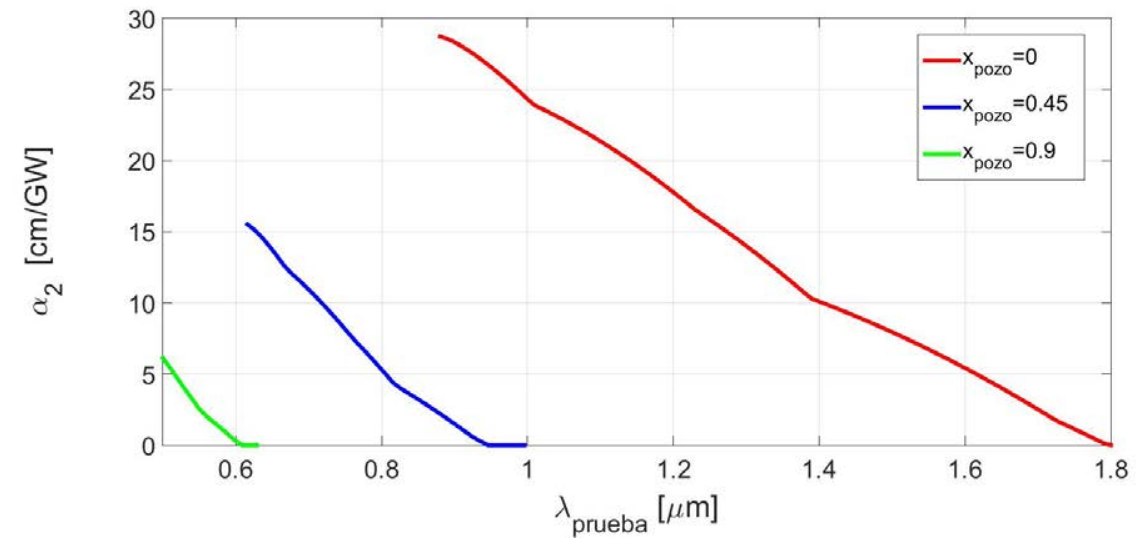

No reportamos las figuras para el caso de un haz de bombeo de $1.310 \mathrm{~nm}$ por ser muy similar a los ya mostrados, solo cambia el valor para la longitud de onda de prueba a la que ocurre la máxima absorción, como $1.310 \mathrm{~nm}$ es una longitud de onda más energética que $1.550 \mathrm{~nm}$ es lógico que la absorción ocurra a longitudes de onda de prueba mayores. Adicionalmente el valor la absorción es levemente menor, por ende, es óptimo usar una longitud de onda de bombeo de $1.550 \mathrm{~nm}$.

\section{CONCLUSIONES}

Las estructuras de múltiples pozos cuánticos poseen opciones de diseño que pueden restringirse, por ejemplo, es importante el ancho del pozo, hay una garantía de mejor 2PA para anchos de pozo que no superes los $20 \mathrm{~nm}$, el límite inferior es determinado por la capacidad de fabricarlas con la tecnología actual; la fracción molar de aluminio dopante es importante porque de ella depende según la Ecuación (2) como cambia la energía necesaria para excitar la absorción; la longitud de onda de bombeo para excitar absorción de dos fotones es justificada ya que se convierte en un parámetro de diseño fuerte a la hora de obtener mayor eficiencia, ya que podemos aprovechar el caso END-2PA. Claramente podemos ver en las Figura 3 y 4 la fuerte dependencia que existe para la absorción de dos fotones el uso de diferentes concentraciones de fracción molar de aluminio, las longitudes de onda de prueba y bombeo además de la polarización.

Estos estudios de simulación abren un panorama para la optimización y posibilidades de un dispositivo funcional para telecomunicaciones ópticas. El uso de la absorción de dos fotones extremadamente no degenerado es la vía mas optima de aplicar a un dispositivo que cumpla características de alta conmutación.

\section{REFERENCIAS}

Adachi, S. (1985). GaAs, AlAs, and AlxGa1-xAs@B: Material parameters for use in research and device applications. J. Appl. Phys 58, pp. R1-R29.

Chuang, S. L. (2009). "Refractive Index of AlGaAs System". En: Physics of Photonic Devices. Segunda edición. New Jersey, Ed Wiley Series in Pure and Applied Optics, 2009. pp. 264-265.

Colace, L; Masini, G; Altieri, A; Assanto, G. (2006). Waveguide photodetectors for the near-infrared in polycrystalline germanium on silicon. IEEE Photon. Technol. Lett 18 (9), pp. 1094-1096.

Fishman, D. A; Cirloganu, C. M; Webster, S; Padilha, L. A; Monroe, M; Hagan, D. J; Van Stryland, E. W. (2011) Sensitive Mid-Infrared Detection in Wide-Bandgap Semiconductors Using Extreme Non-Degenerate Two Photon Absortion. Nat Photonics 5, pp. 561565. 
Ren, S; Rong, Y; Claussen, S.A; Schaevitz, R.K; Kamins, T.I; Harris, J.S; Miller, D. A. B. (2011). Thin dielectric spacer for the monolithic integratioun of bulk germanium or germanium quantum wells with siliconon-insulator waveguides. IEEE Photon. J 3 (4), pp. 739-747.

Pattanaik, H. S; Reichert, M; Khurgin, J. B; Hagan, D. J; Van Stryland, E. W. (2016). Enhancement of Two-Photon Absortion in Quantum Wells for Extremely Nondegenerate Photon Pairs. IEEE J Quantum Elect 52.

Villeneuve, A; Kang, J. U; Aitchison, J. S; Stegeman, G. I. (1995). Unity ratio of cross to selfphase modulation in bulk AlGaAs and AlGaAs/GaAs multiple quantum well waveguides at half the band gap. App. Phy. Lett 67, pp. 760-762.

Wagner, S. J; Meier, J; Helmy, A. S; Aitchison, J. S; Sorel, M; Hutchings, D. C. (2007) Polarization-dependent nonlinear refraction and two-photon absorption in GaAs/AlAs superlattice waveguides below the halfbandgap, J. Opt. Soc. Am. B 24, pp. 1557-1563.

Xia, C; Spector, H.N. (2009). Nonlinear Franz-Keldysh effect: Two photon absorption in semiconducting quantum wires and quantum boxes. Journal of Applied Physics 106(124302), pp. 1-6.

\section{PARA CITAR ESTE ARTÍCULO TO REFERENCE THIS ARTICLE / PARA CITAR ESTE ARTIGO /}

Álvarez Ocampo, C.A. (2017). Absorción de dos fotones en pozos cuánticos formados con GaAs dopado con Aluminio. Revista EIA, 14(28), julio-diciembre, pp. 77-83. [Online]. Disponible en: https://doi.org/10.24050/reia.v14i28.1128 